International Journal of Soft Computing 8 (2): 82-86, 2013

ISSN: 1816-9503

(C) Medwell Journals, 2013

\title{
Retrecheck: An Efficient Handoff and Buffer Ranging Framework to Minimize Packet Loss Rate Through Check Point Retransmission Using 802.16m WiMAX Networks
}

\author{
${ }^{1}$ D. Karunkuzhali and ${ }^{2}$ D.C. Tomar \\ ${ }^{1}$ Department of CSE, Sathyabama University, Chennai, India \\ ${ }^{2}$ Department of CSE, Misrimal Navajee Munoth Jain Engineering College, \\ Chennai, Tamil-Nadu, India
}

\begin{abstract}
This study meant for the buffer management and to reduce packet loss during WiMax communication where the networking involves distress regarding buffer range and traffic management. Resaechers evident that Retrecheck $802.16 \mathrm{~m}$ based network frames efficient buffer management with effort from base station scheduler and subscriber station scheduler. These processes incur least bandwidth under utilisation thereby reducing the transmission delay. All these domains were put forth through Admission Control (AC) mechanism and a Dynamic Buffer Allocation (DBA) process which directly clears packet sizing buffer ranging with accession from check point constraints where the left packets will be put into transmission thereby it gives effective buffer management system with improved handoff standards between the sender base station and subscriber base station.
\end{abstract}

$\underline{\text { Key words: Transmission delay, handoff, check point, buffer range, DBA }}$

\section{INTRODUCTION}

In wired LAN based network fixed buffers are used. This increases the round trip time delay for queuing data buffer for sending and receiving data. Delay may occur due to processing data (Shrestha et al., 2009). If fixed buffer size are used. To over come this, the buffer size must be altered dynamically based on the bandwidth (Pontes et al., 2008; Zhag, 2007; Rhee et al., 2011). This reduces the overall round trip time delay of the host connected in a network. Some traditional techniques for available bandwidth use throughput to provide a coarse estimate of bandwidth. The bandwidth available estimate is directly related to the throughput that the sender is willing to test at any instant. On a packet loss, this mechanism provides a loose upper bound for the available bandwidth. Packet loss is actually a better estimate of buffer capacities in the network than of available bandwidth additionally given the relatively low costs associated with the deployment of a WiMAX 802.16 network (Sankarasubramaniam and Subramanina, 2012; Li et al., 2007; Sengar et al., 2011) it is now economically viable to provide last-mile broadband Internet access in remote locations.

WiMAX 802.16 was a replacement candidate for cellular phone technologies such as GSM and CDMA or can be used as an overlay to increase capacity. Fixed WiMAX 802.16 is also considered as a wireless backhaul technology for second generation, third generation and forth generation networks in both developed and developing nations.

This study mainly concentrates on the buffer sizing which is to dynamically alter its range in comparison with receiver packet size and with the cut-off packet size. And the interoperability for the medium access control static buffer mobile subscriber station and base stations are directly altered through the proposed methodology called Retrecheck.

In most cities backhaul for urban operations is typically provided via one or more copper wire line connections whereas remote cellular operations are sometimes backhauled via satellite. An integrated Wi-Fi access point provide the WiMAX Internet connectivity to multiple devices throughout the home or business and Ethernet ports to connect directly to a computer or DVR instead. One or two analog telephone jacks to connect a land-line phone and take advantage of VoIP. In other regions, urban and rural backhaul is usually provided by microwave links (the exception to this is where the network is operated by an incumbent with ready access to the copper network). WiMAX $802.16 \mathrm{~m}$ has more substantial backhaul bandwidth requirements than legacy cellular applications. Consequently the use of wireless microwave backhaul is on the rise in North America and existing micro wave backhaul links in all regions are being upgraded. Capacities of between $34 \mathrm{Mbit} \mathrm{sec}^{-1}$ and

Corresponding Author: D. Karunkuzhali, Department of CSE, Sathyabama University, Chennai, India 
$1 \mathrm{Gbit} \mathrm{sec}^{-1}$ are routinely being deployed with latencies in the order of $1 \mathrm{~m} \mathrm{sec}$. In many cases, operators are aggregating sites using wireless technology and then presenting traffic on to fiber networks where convenient thereby introducing ways to transmit packets from where the packet loss is initiated or in other terms where the packet transmission is broken.

\section{RELATED WORK}

The standard $802.16 \mathrm{~m}$ is improved with its movement and transaction compatibilities with its prior work. Handoff between WiMax base stations will be considered as a series of events. The transaction and the access point for the reselection process is a periodic in which the mobile node within the cell where the base station is available. This cell provides the information to which target base station the packet should be sent. The original handoff begins at the decision which starts at the main subscriber station, the serving base station base or on the cell. Handoff follows with the initiation of mobile subscriber the flow is given with entire process of handoff (Pontes et al., 2008; Zhag, 2007; Injong et al., 2011). A unique identity in the phase of this procedure a prominent phase of the $\mathrm{HO}$ procedure is learning and knowledge acquisition phase where mobile base station is used by the cell to discover neighbouring base station. At the time when the cell space is capable enough the form a sequence of base stations to construct unit of base stations the mobile buffer size is replaced with that of the packet size. If transmission in subjected to loss or failure occurs, the information maintained at the check point and the base station will help the system for retransmission of packets across the network with dynamic buffer ranges. The next handoff procedure is scanning followed by actual handoff. And the exchange of control messages between service BS and target BSs. The decision process starts with the selection of target base station thereby the next or successor base station list will be maintained alone with that of the targets base station association list.

This list will then scanned by the main system with the time factor in the packet is to be sent if the time of transaction follows possible parameters the process flows to sending of packets to the target base station with that of the address of target from the list, if not it will subjected to scanning process again. Then, comes the operation of receivers initial handoff ranging response report from servers base station and the ranging of buffer is specified followed by the packet transmission the once the packet is received thoroughly the range values were set for valid time. In other case where the transmission is lost the buffer will be altered according to the size of packet that to be sent. Next comes the process of post handoff decision functions, it begins with scanning of signal strength where the capacity of bandwidth is subjected to test of modification.

Selection of target base station is to be served at the mobile station which makes it possible start the time of transaction. Once the target base station is ready to accept the packets with the initial buffer range the transmission is started. At the time when the time exceeds the limit connection is broken which makes buffer ranging parameters filled. In case of valid parameter, the connection is established with the retransmission acknowledgement provides data transfer.

\section{THE METHODOLOGY}

In the proposed system the process of handing off is enhanced in the system with effort from Retrecheck which is $802.16 \mathrm{e}$ WiMax standard in addition to check points where the retransmission works are carried out through the efficient allocation of space of bandwidth with remaining packets that are blocked or subjected to failure in communication errors. The following explanation makes it clear for the whole process of the proposal where it starts with handoff operation in WiMax. Admission control systems in the Retrecheck allow noncompliant applications to be denied access, placed in a quarantined area or given restricted access to computing resources thus keeping insecure nodes from infecting the network. This admission control in $802.16 \mathrm{~m}$ allows the unauthorized application not to enter into the system of packet transmission and this whole system is shown in Fig. 1.

Parallel to this buffer manager will concentrates on the size of buffer in sending base station and the target base station. This process gets input from the acknowledgement whether positive if the transmission accomplished or negative is there is failure in transmission rate.

Dynamic buffer sizing is implemented to achieve time delay reduction during retransmission. In the system, we desire dynamic duffer ranging instead of reducing the congestion window, this can be initiated by monitoring the bandwidth. Based on the bandwidth along with the packet loss hence researchers can dynamically increase or decrease the buffer size. This avoids the unnecessary delay in RTT when congestion not occurred results in efficient usage of buffer. The date access and sending is done faster than before. This process leads the system for efficient through put of packet transmission exact utilisation of system resources, i.e., buffer size and bandwidth, proper bandwidth utilisation.

Once the admission control process in Retrecheck rejects the incoming process it will be sent to check point 


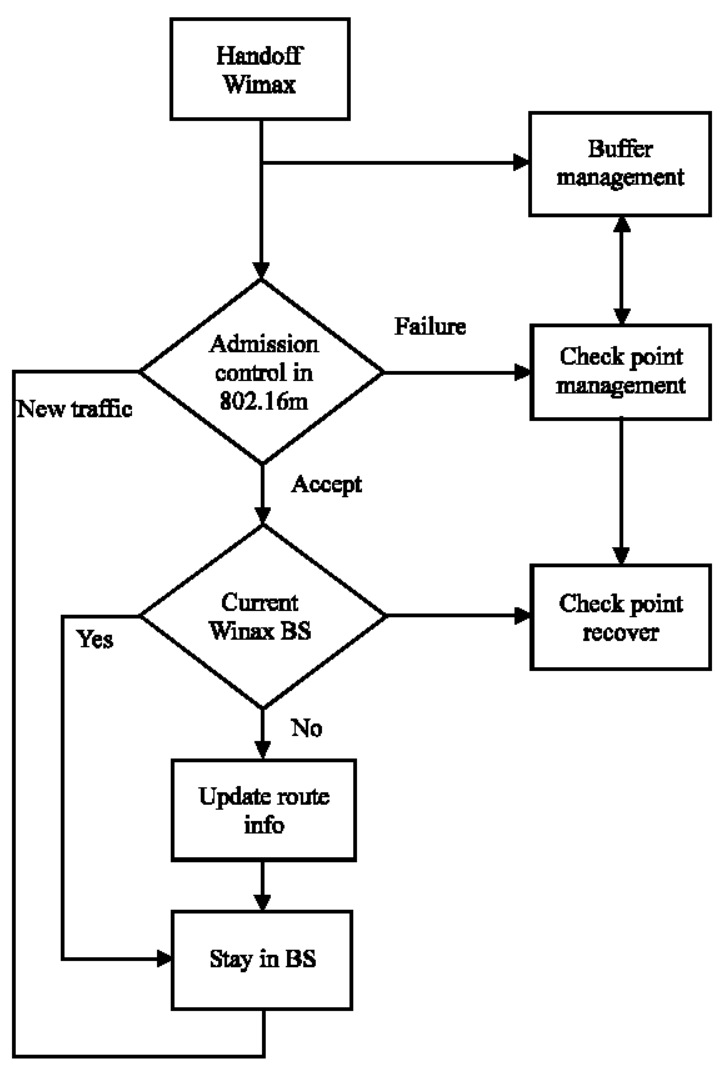

Fig. 1: Retrecheck handoff $802.16 \mathrm{~m}$ frame work

which is a point in time as of which the state of all the packets in the system will be preserved. When the system needs retransmission, it is initialized to the state at a demarcation event. In other terms, this functionality of check point comes to consideration when the transmission fails where the information about the number of packets that are sent already and the packet to be sent will be monitored. The time value when the demarcation happens, i.e., transmission fails check point makes its way through the data it collected once negative acknowledgement is received analysis is made for check point recovery. This can be explained by considering a transmission of elementary packets which are 100 in number and during the transmission if the failure occurs at the time value measure at 80 th packet the check point clearly holds the number and retransmission will be made with the contingency sequence of packet from where the flow is blocked.

When the admission control mechanism accepts the flow is subjected exclusively to current WiMax base station. From here the handoff will be directed to two cases if WiMax base station is capable of accepting the incoming packets with respect to its size. The ranging is done in the base station's resource allocator which intern referred as buffer manager and stays in the base station subsystem. In extreme case of improper channel of bandwidth allocation the entire routing path will be examined for maximum possible optimal routing process and this route is updated in the already existing information regarding transmission control parameters like time and check points. Thus, efficient routing along with improved handoff is achieved by the way of reducing time delay and packet loss during sending and receiving process at both the base stations thereby researchers can increase throughput of packet transmission.

\section{SYSTEM IMPLEMENTATION}

The proposed system Retrecheck is having the transmission control is implemented in terms of above mentioned $802.16 \mathrm{~m}$ with handoff standards. The following flow will show the entire process in terms of codes with vital admission control mechanism, buffer management and check point management.

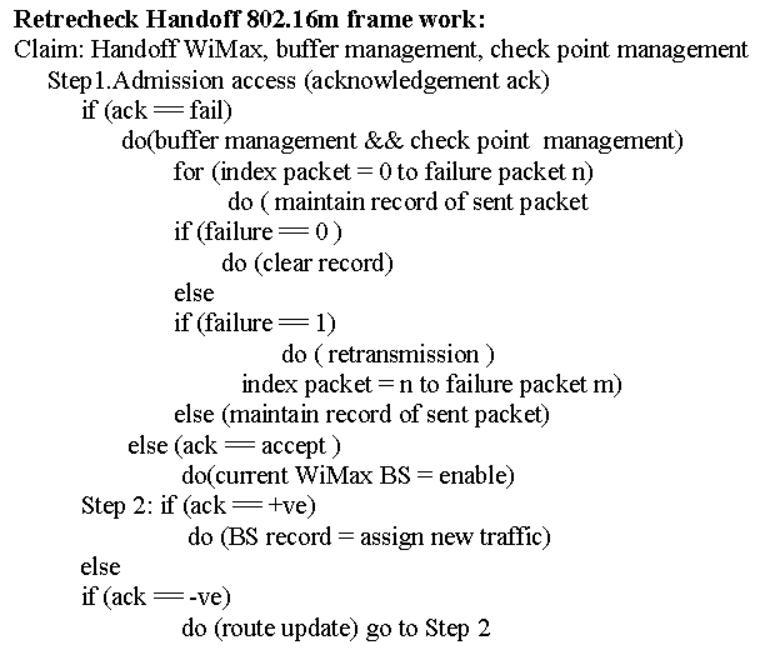

\section{EXPERIMENTAL STUDY}

The conceptual arguments are put forth to prove efficiency standards in $801.16 \mathrm{~m}$ is exponent when compared to the predecessors. Researchers have presented more accuracy in terms of efficiency statistically. The consideration is made in order to show the advancement of every parameter like time, throughput, bandwidth, PDR, packet delay, admission blocking probabilities and packet loss rate.

In the proposed system Retrecheck during the data transmission the exactness is calculated with that of number of packets receiver in the mean interval of time the followinggraphs showing green line indicates the analysis resultant of $802.16 \mathrm{~m}$ with handoff and the red line showing the measurement of earlier 802.16e standard. 


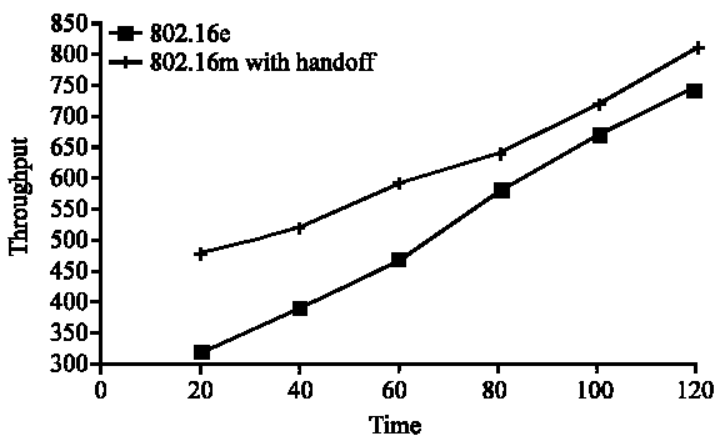

Fig. 2: Time vs. Avg. throughput

When the time measurement is at equal interval of seconds in both the cases and the throughput in the communication networks like 802.16 is the average rate of successful data delivery over a communication channel. This data may be delivered over a physical or logical link or pass through a certain network node. The throughput is usually measured in bits per second (bit/sec or bps) and sometimes in data packets per second or data packets per time slot. This slot is drastically improved with a variance rate of more the 150 packets and shown in Fig. 2.

The bandwidth though which the range within a band of frequencies or wavelengths is taken into consideration of our next test case where it is compared with packet delay rate. The amount of data that can be transmitted in a fixed amount of time is measured. And number of packet transmitted in that band is considered when packet loss occurs and the bandwidth is usually expressed in bits per second (bps) or bytes per second. For the system, the bandwidth is expressed in cycles per second or Hertz $(\mathrm{Hz})$. Due to imprecise handoff and dynamic buffering in both sides of base station the packet delayed rate is improved with intelligent check point management. Figure 3 shows incredible declination of packet delay with respect to bandwidth allocated. The bandwidth is particularly important for $\mathrm{I} / \mathrm{O}$ devices. For example, a fast disk drive can be hampered by a bus with a low bandwidth. In Fig. 4, researchers have shown the increase of efficiency in terms of packet delay with respect to time factor again. In earlier standard there is a gradual hike in the delay as the time increases incurring the conclusion that when the system begins its transaction it has least delay and when the traffic increases (time) the delay increases form direct proportionality. In the standard packet delay is not linear researchers have slight deviations and minimum delay because of dynamic buffering and periodic update of routing information that gives optimal routing path. Through admission blocking probability the handoff is subjected to current WiMax base station and target base

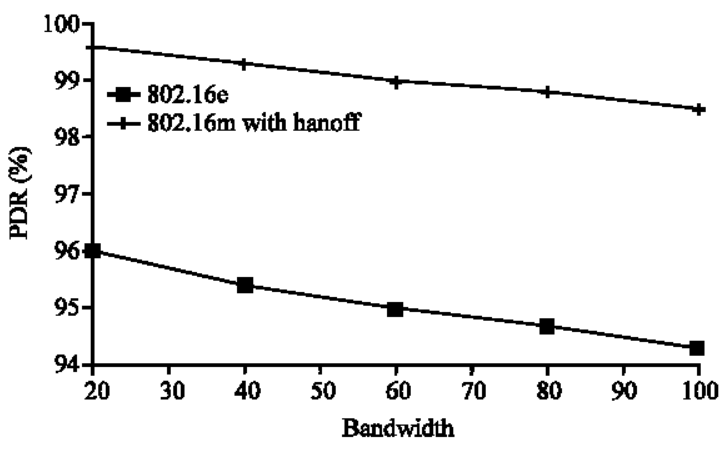

Fig. 3: Bandwidth vs. PDR

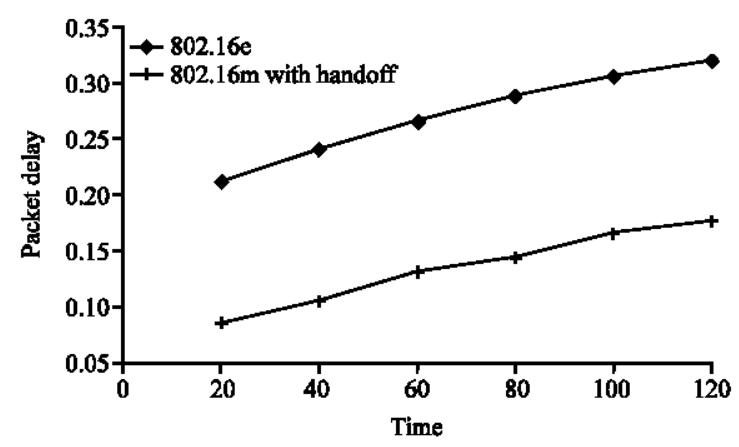

Fig. 4: Time vs. packet delay

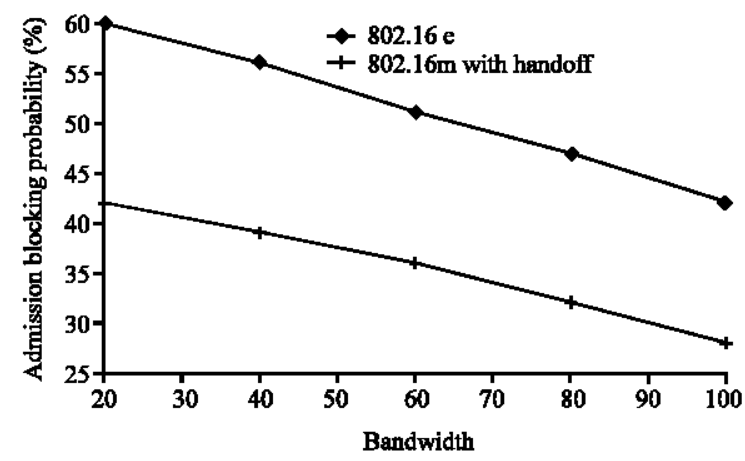

Fig. 5: Bandwidth vs. admission blocking probability

station reducing the probability $>20 \%$ is shown in Fig. 5 . Eventually Fig. 6 indicates the vital packet losspacket loss rate is taken into account with respect to bandwidth allocation showing that the rate of packet loss is directly proportional to size of bandwidth utility.

All these works of experimental studies were carried out with NS-2 where the WiMax standards have verified through code and the parameters of the resultant graph is extracted through NS-2. The area of operation in WiMax handoffs and base station analysis are made under coverage region of $1500 \times 300$ consisting of 25 mobile hosts and their buffer ranging parameters with respect to packet size sent between those mobile base stations. 


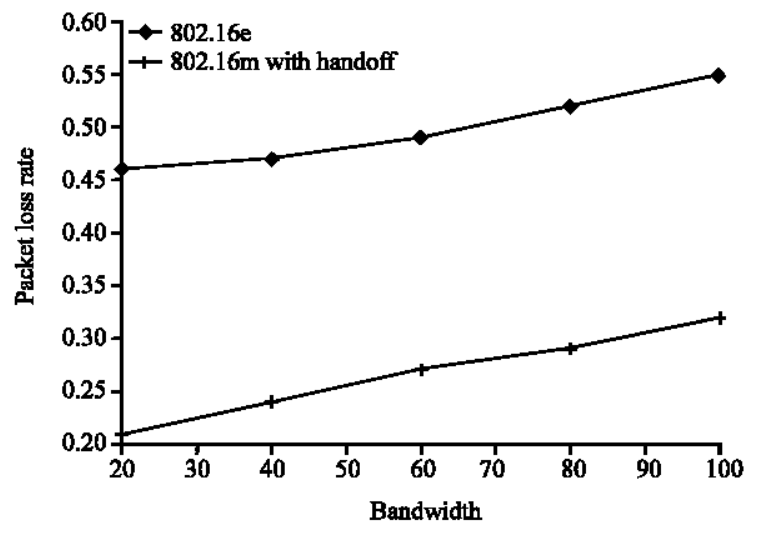

Fig. 6: Bandwidth utility vs. packet loss rate

Thus, all the values which are necessary for the study is collected and simulation is made according to the outcome for the experiment and accuracy in performance evolution is given evidently.

\section{CONCLUSION}

Retrecheck $802.16 \mathrm{~m}$ delivers elevated liveliness of bandwidth across the transmission medium with efforts from $802.16 \mathrm{~m}$ handoff and check point mechanism. Hence, researchers can achieve dynamic buffer ranging for the failure recovery process through mechanism of check point. This suggests that the proposed model and utilization of optimization routing may be useful in terms of dynamically altering buffer ranging and handoff thereby increasing profitability of increased throughput, efficient usage of buffer, reduced overall round trip time delay and dynamically changing buffer size based on the bandwidth according to specific quality of service constraints.

\section{REFERENCES}

Li, B., Q. Yang, P.L. Chor and L. G. Choon, 2007. A survey on mobile WiMAX [wireless broadband access]. Commun. Magazines, 45: 70-75.

Pontes, A., D. dos Passos Silva, J. Jailton, O. Rodrigues and K.L. Dias, 2008. Handover management in integrated WLAN and mobile WiMAX networks. IEEE Wireless Commun., 15: 86-95.

Rhee, I., M. Shin, S. Hong, K. Lee, S. J. Kim and S. Chong, 2011. On the levy-walk nature of human mobility. IEEE/ACM Trans. Network., 19: 630-643.

Sankarasubramaniam, K. and S. Subramanina, 2012. A performance study of uplink scheduling in WiMAX network. Proceedings of 2nd International Conference on ICRTIT, April 19-21, 2012, Madras Institute of Technology, Anna University, Chennai, pp: 377-382.

Sengar, S.S., N. Tyagi and A. P. Singh, 2011. A Survey on WiMAX-3G inter working. Proceedings of $3 \mathrm{rd}$ International Conference on ICCSN, May 27-29, 2011, Xian, China, pp: 54-58.

Shrestha, S. L., N. O. Song and S.Chong, 2009. Seamless realtime traffic handover policy for IEEE $802.16 \mathrm{~m}$ mobile WiMAX. Proceedings of 43rd Annual Conference on Information Sciences and Systems, March 18-20, 2009, The John Hopkins University, Baltimore, MD., USA., pp: 252-257.

Zhag, B., 2007. Buffer management for WiMax/802.16 subscriber stations. M.Sc. Thesis, Carleton University. 